\title{
A formal enantioselective synthesis of $(+)$-dodoneine via cyclic sulfate methodology
}

\author{
Srinivasu V.N. Vuppalapati, Siddamal R. Putapatri, and Srinivas Kantevari* \\ Organic Chemistry Division-II, Indian Institute of Chemical Technology, Hyderabad-500007, \\ INDIA \\ E-mail: kantevari@yahoo.com, kantevari@gmail.com
}

\begin{abstract}
An enantioselective formal synthesis of $(+)$-dodoneine is described using the Sharpless asymmetric dihydroxylation and regioselective nucleophilic opening of cyclic sulfate as the key steps.
\end{abstract}

Keywords: Sharpless asymmetric dihydroxylation, dodoneine, cyclic sulfate methodology, enantioselective synthesis

\section{Introduction}

Dodoneine 1, [(R)-6-[(S)-2-hydroxy-4-(4-hydroxyphenyl)butyl]-5,6-dihydropyran-2-one], is a recently isolated $\alpha, \beta$-unsaturated $\delta$-lactone from the methanolic extract of a plant hemi parasite, Tapinanthus dodoneifolius DC Danser (also known as African mistletoe) found on a sheanut tree in Loumbila, West Africa. ${ }^{1}$ The structure of 1 was determined from spectroscopic and X-ray crystallographic analysis of the camphorsulphonate derivative of dodoneine.

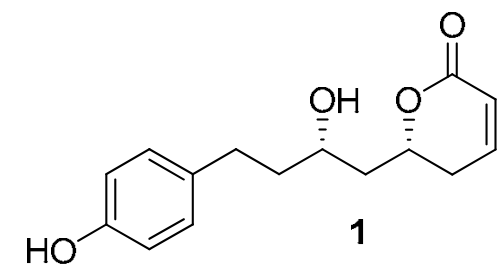

The compound (+)-Dodoneine 1 exhibited relaxing effect on preconstricted rat arotic rings. The unique as well as challenging structural feature of this class of compounds along with their potential biological activity has aroused great interest among synthetic organic and medicinal chemists. $^{2}$ 
The synthesis approaches described till now in the literature for dodoneine 1 involve 1) enantioselective addition of allyl metal reagents to aldehydes, followed by Grubbs ring closing metathesis $^{3}$ 2) Horner-Wadsworth-Emmons olefination and Crimmins aldol approach ${ }^{4}$ and 3 ) Sharpless asymmetric epoxidation followed by 1,3-syn diasteroselective reduction and Grubbs ring closing metathesis. ${ }^{5}$ As part of our research work aimed at developing enantioselective syntheses of naturally occurring lactones, the Sharpless asymmetric dihydroxylation and subsequent transformation of the diols formed via cyclic sulfites/ sulfates were envisaged as powerful tools offering considerable opportunities for synthetic manipulations. ${ }^{6}$ Herein we report a new and highly enantioselective formal synthesis of $(+)$-dodoneine employing the Sharpless asymmetric dihydroxylation as the source of chirality.

\section{Results and Discussion}

Our approach for the synthesis of $(+)$-dodoneine was envisioned via the retrosynthetic route shown in Scheme 1.
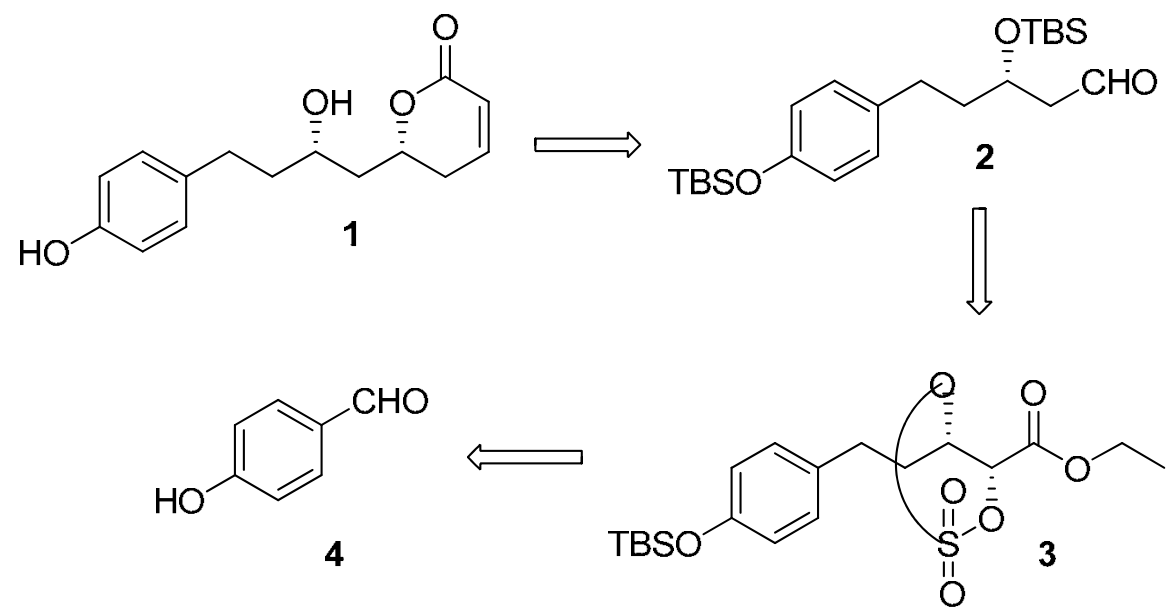

Scheme 1. Retrosynthetic route to $(+)$-dodoneine.

The key intermediate aldehyde 2 was visualized as an ultimate precursor for the target molecule, which could be obtained from the hydride opening of cyclic sulfate $\mathbf{3}$ and subsequent hydrolysis. The cyclic sulfate 3 could be derived from commercially available $p$-hydroxy benzaldehyde 4 through series of reactions comprising double $2 \mathrm{C}$ Wittig olefinations and Sharpless asymmetric dihydroxylation. The salient feature of our synthetic strategy was on the presumption that regioselective nucleophilic opening of cyclic sulfate would occur at the $\alpha$ carbon. The detailed route for the synthesis of aldehyde 2 with reagents and reaction conditions is outlined in Scheme 2. 
The synthesis was initiated with TBS protection (TBS-Cl, imidazole, $\mathrm{CH}_{2} \mathrm{Cl}_{2}, 0{ }^{\circ} \mathrm{C}$ to room temperature, $1.5 \mathrm{~h}, 96 \%$ yield) of commercially available $p$-hydroxybenzaldehyde 4 , followed by standard $2 \mathrm{C}$ Wittig olefination with $\mathrm{Ph}_{3} \mathrm{PCHCOOEt}$ in benzene under reflux conditions to give trans-olefin 5 in $92 \%$ yield. Reduction of trans-olefin ester 5 using $\mathrm{LiAlH}_{4}$ at $25{ }^{\circ} \mathrm{C}$ gave mixture of products along with low yield of desired alcohol $\mathbf{6}$. In order to obtain the best yield of alcohol 6, a two step procedure was devised involving catalytic hydrogenation of double bond using $10 \%$ $\mathrm{Pd} / \mathrm{C}, \mathrm{H}_{2}$ (92\% yield); followed by $\mathrm{LiAlH}_{4}$ reduction of ester to alcohol 6 in dry THF at $0{ }^{\circ} \mathrm{C}$ (91\% yield). Swern oxidation of alcohol 6 and subsequent 2C Wittig reaction with (ethoxycarbonylmethylene)triphenylphosphorane in benzene under reflux conditions<smiles>CC#CCOC(=O)C=Cc1ccc(OCC#CC)cc1</smiles>

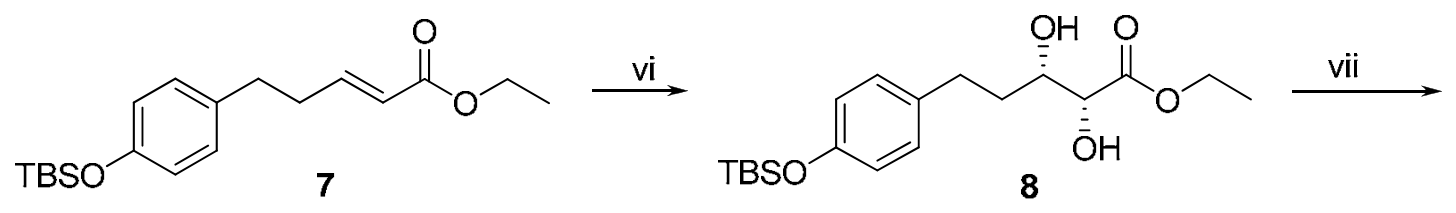

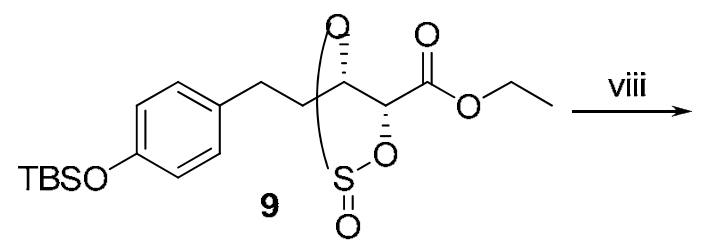

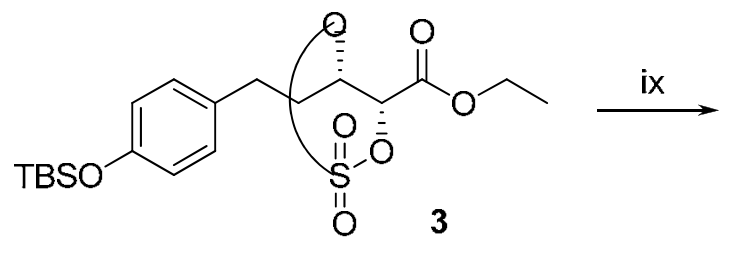<smiles>[R15]Oc1ccc(CC[C@H](O)CC(=O)OCCC([X])C)cc1</smiles>

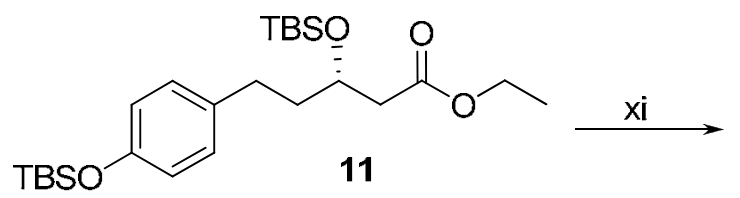<smiles>CC#CCCCCC(CC=O)O[SnH3]</smiles>

Scheme 2. Reagents and conditions: (i) TBSCl, imidazole, $\mathrm{CH}_{2} \mathrm{Cl}_{2}, 0{ }^{\circ} \mathrm{C}, 1.5 \mathrm{~h}, 96 \%$; (ii) $\mathrm{Ph}_{3} \mathrm{PCHCO}_{2} \mathrm{Et}$, Benzene, reflux, $6.5 \mathrm{~h}, 92 \%$; (iii) (a) $10 \% \mathrm{Pd} / \mathrm{C}, \mathrm{H}_{2}$ (1atm), EtOAC, overnight, 92\%; (b) $\mathrm{LiAlH}_{4}, \mathrm{THF}, 0{ }^{\circ} \mathrm{C}, 1.5 \mathrm{~h}, 91 \%$; (iv) $(\mathrm{COCl})_{2}$, DMSO, $\mathrm{Et}_{3} \mathrm{~N}, \mathrm{CH}_{2} \mathrm{Cl}_{2},-78{ }^{\circ} \mathrm{C}, 30 \mathrm{~min}$, 94\%; (v) $\mathrm{Ph}_{3} \mathrm{PCHCO}_{2} \mathrm{Et}$, benzene, reflux, 8 h, 91\%; (vi) AD-mix- $\alpha, \mathrm{MeSO}_{2} \mathrm{NH}_{2}$, t-BuOH-H $\mathrm{H}_{2} \mathrm{O}$ (1:1), $0{ }^{\circ} \mathrm{C}$, Overnight, $85 \%$; (vii) $\mathrm{SOCl}_{2}, \mathrm{Et}_{3} \mathrm{~N}, \mathrm{CH}_{2} \mathrm{Cl}_{2}, 0{ }^{\circ} \mathrm{C}, 30 \mathrm{~min}, 94 \%$; (viii) $\mathrm{RuCl}_{3}, \mathrm{NaIO}_{4}$, $\mathrm{CCl}_{4}-\mathrm{MeCN}_{-} \mathrm{H}_{2} \mathrm{O}: 2: 2: 3,0{ }^{\circ} \mathrm{C}, 30 \mathrm{~min}, 92 \%$; (ix) $\mathrm{NaBH}_{4}$, DMF, 15 min, then THF, cat. Conc. $\mathrm{H}_{2} \mathrm{SO}_{4}$, cat. $\mathrm{H}_{2} \mathrm{O}, 20$ min, $90 \%$; (x) TBSCl, imidazole, $\mathrm{CH}_{2} \mathrm{Cl}_{2}, 0-25{ }^{\circ} \mathrm{C}$ overnight, $95 \%$; (xi) DIBAL-H, $\mathrm{CH}_{2} \mathrm{Cl}_{2},-78{ }^{\circ} \mathrm{C}, 10 \mathrm{~min}, 88 \%$. 
furnished $\alpha, \beta$-unsaturated ester 7 in good yields (91\%). The dihydroxylation of trans olefin ester 7 with AD-mix- $\alpha$, methane sulfonamide in tert-BuOH- $\mathrm{H}_{2} \mathrm{O}(1: 1)$ under the Sharpless asymmetric dihydroxylation reaction conditions gave diol 8 in $85 \%$ yield and $96 \%$ ee (from ${ }^{1} \mathrm{H}$ NMR analysis of its diacetate using Eu(III) chiral shift reagent). Sharpless and co-workers have also observed $^{6 a, 7}$ that vicinal diol cyclic sulfates are "like epoxides only more reactive". With this clue, the diol 8 was treated with thionyl chloride in the presence of triethyl amine in dichloromethane at $0{ }^{\circ} \mathrm{C}$ to give isomeric cyclic sulfite 9 in $94 \%$ yield. Ruthenium chloridesodium periodate oxidation of sulfite 9 gave cyclic sulfate 3 in $92 \%$ yield. The essential feature of our synthetic strategy shown in the Scheme 2 was observed on the presumption that the nucleophilic opening of the cyclic sulfate would occur in the regiospecific manner at the $\alpha$ position. Reduction of cyclic sulfates to mono alcohols with sodium borohydride in dimethyl acetamide was originally recommended. ${ }^{7 \mathrm{a}}$ The intermediate sulfate esters were then hydrolyzed in a $20 \%$ aqueous $\mathrm{H}_{2} \mathrm{SO}_{4}$-ether system. Alternatively, hydrolysis could be affected with a catalytic amount of concentrated sulfuric acid and 0.5-1.0 equiv of water in THF. ${ }^{8}$

We have observed that, hydrolysis (with catalytic amount of sulfuric acid and water) of intermediate sulfate ester, obtained from cyclic sulfate 3 after treatment with sodium borohydride in DMF at $25^{\circ} \mathrm{C}$, was sluggish and gave the desired product alcohol 10 in low yields $(<10 \%)$. Conducting both the stages of reaction at $0{ }^{\circ} \mathrm{C}$ for longer hours $(2-3 \mathrm{~h}$ each) improved the formation of alcohol 10 (52\% yield), but $\alpha, \beta$-unsaturated ester 7 was also obtained as side product in substantial amount $(\sim 35 \%)$. After series of experiments, we have established that treatment of cyclic sulfate 3 with sodium borohydride in DMF for 15 minutes at $0{ }^{\circ} \mathrm{C}$, followed by hydrolysis with catalytic amount of concentrated sulfuric acid and 0.35 equiv of water in THF at $0{ }^{\circ} \mathrm{C}$ for $20 \mathrm{~min}$ afforded the desired alcohol 10 in quantitative yield (90\%). The choice of reaction conditions (reaction temperature and time) is crucial for the successful yield of the desired product alcohol 10.

Next, the secondary hydroxyl group of compound $\mathbf{1 0}$ was protected (TBS-Cl, imidazole, $\mathrm{CH}_{2} \mathrm{Cl}_{2}$, overnight) to furnish TBS protected ester $\mathbf{1 1}$ in $95 \%$ yield. The ester $\mathbf{1 1}$ was then subjected to reduction with DIBAL-H in dichloromethane at $-78{ }^{\circ} \mathrm{C}$ to yield aldehyde 2 in $88 \%$ yield. Absolute stereo chemistry of the product aldehyde (S)-2 was ascertained by comparing all the analytical (specific rotation, $\left.[\alpha]_{\mathrm{D}}{ }^{25}=+5.54\left(\mathrm{c} 2.1, \mathrm{CHCl}_{3}\right), \mathrm{Lit}^{3 \mathrm{c}}[\alpha]_{\mathrm{D}}{ }^{25}=+5.57\right)$ and ${ }^{1} \mathrm{H}$ and ${ }^{13} \mathrm{C}$ NMR spectral data with recently reported literature values. ${ }^{3 \mathrm{c}}$

Natural product $(+)$-Dodoneine 1 was finally obtained from aldehyde $(\boldsymbol{S})-\mathbf{2}$ by following a well described $^{3,5}$ reaction sequence comprising asymmetric allylation with allyl magnesium bromide, acylation with acryloyl chloride and Grubbs ring closing metathesis.

In conclusion, a formal synthesis of $(+)$-dodoneine $\mathbf{1}$ has been accomplished by Sharpless asymmetric dihydroxylation and regioselective nucleophilic opening of cyclic sulfate. The optimum conditions for the key steps, nucleophilic opening of cyclic sulfate 3 with sodium borohydride in regioselective manner at the $\alpha$-position $\left(0{ }^{\circ} \mathrm{C} / 15 \mathrm{~min}\right)$ and subsequent hydrolysis of sulfate ester $\left(0{ }^{\circ} \mathrm{C} / 20 \mathrm{~min}\right)$ is ascertained after series of experimentations. Further application of 
this cyclic sulfate methodology to the synthesis of biologically active compounds for structureactivity studies is currently underway in our laboratory.

\section{Experimental Section}

General. All the reactions were monitored by TLC (recoated silica plates and visualizing under UV light). Air-sensitive reagents were transferred by syringe or double-ended needle. Evaporation of solvents was performed at reduced pressure on a Buchi rotary evaporator. ${ }^{1} \mathrm{H}$ and ${ }^{13} \mathrm{C}$ NMR spectra of samples in $\mathrm{CDCl}_{3}$ were recorded on Bruker UXNMR FT-300 MHz (Avance) spectrometer and Varian FT-500MHz (Inova). Chemical shift reported are relative to an internal standard TMS $(\delta=0.0)$. Mass spectra were recorded in EI conditions at $70 \mathrm{eV}$ on an LC-MSD (Agilent technologies) spectrometer. All high-resolution spectra were recorded on QSTAR XL hybrid MS/MS system (Applied Biosystems/ MDS sciex, Foster City, USA), equipped with an ESI source (IICT, Hyderabad). Column chromatography was performed on silica gel (60-120 mesh) supplied by Acme Chemical Co., India. TLC was performed on Merck 60 F-254 silica gel plates. Optical rotations were measured with JASCO DIP-370 Polarimeter at $25{ }^{0} \mathrm{C}$. Commercially available anhydrous solvents $\mathrm{CH}_{2} \mathrm{Cl}_{2}$, THF, and EtOAc were used as such without further purification.

\section{4-(tert-Butyldimethylsilyloxy)benzaldehyde}

To the stirred solution of $p$-hydroxybenzaldehyde $4(6.0 \mathrm{~g}, 49.18 \mathrm{mmol})$ in anhydrous $\mathrm{CH}_{2} \mathrm{Cl}_{2}$ $(35 \mathrm{~mL})$, imidazole $(5.0 \mathrm{~g}, 73.77 \mathrm{mmol})$ followed by tert-butyldimethylsilyl chloride $(8.89 \mathrm{~g}$, $59.01 \mathrm{mmol}$ ) were added at $0{ }^{0} \mathrm{C}$ under $\mathrm{N}_{2}$. After stirring the reaction mixture for $1.5 \mathrm{~h}$, at $25{ }^{0} \mathrm{C}$, it was quenched with a saturated aqueous solution of $\mathrm{NH}_{4} \mathrm{Cl}$ and extracted with $\mathrm{CH}_{2} \mathrm{Cl}_{2}(6 \times 30$ $\mathrm{mL}$ ). The combined organic extracts were washed with brine, dried over anhydrous $\mathrm{Na}_{2} \mathrm{SO}_{4}$ and concentrated in vacuum. The residue was purified by flash column chromatography over silica gel (ethyl acetate/hexane, 5:95) afforded 4-(tert-butyldimethylsilyloxy)benzaldehyde (11.12 g, 96\%) as colorless liquid. IR (neat): 2934, 2858, 1697, 1598, 1508, 1272, 1156, $908 \mathrm{~cm}^{-1}$.

${ }^{1} \mathrm{H}$ NMR $\left(300 \mathrm{MHz}, \mathrm{CDCl}_{3}\right): \delta 9.86(\mathrm{~s}, 1 \mathrm{H}), 7.74(\mathrm{~d}, J=7.8 \mathrm{~Hz}, 2 \mathrm{H}), 6.89$ (d, J=8.7Hz, 2H), 0.99 (s, 9H), $0.24(\mathrm{~s}, 6 \mathrm{H}) .{ }^{13} \mathrm{C} \mathrm{NMR}\left(75 \mathrm{MHz}, \mathrm{CDCl}_{3}\right): \delta 189.8,161.2,131.8,120.4,96.2,25.7,18.4$, 4.1. ESI-MS: m/z 259 [M+Na] $]^{+}$HRMS (ESI) $[\mathrm{M}+\mathrm{Na}]^{+} \mathrm{m} / \mathrm{z}$ Calcd for $\mathrm{C}_{13} \mathrm{H}_{20} \mathrm{O}_{2} \mathrm{NaSi}$ : 259.1130; found: 259.1125 .

(E)-Ethyl 3-[4-(tert-butyldimethylsilyloxy)phenyl] acrylate (5). Ethyl (triphenylphosphornylidene)acetate (18.2 g, $54.0 \mathrm{mmol}$ ) was added to a stirred solution of 4-(tert-butyldimethylsilyloxy)benzaldehyde ( $8.5 \mathrm{~g}, 36.0$ $\mathrm{mmol})$ in dry benzene $(80 \mathrm{~mL})$. The reaction mixture was refluxed for $6.5 \mathrm{~h}$, and concentrated in vacuum. Crude residue thus obtained was purified by column chromatography (ethyl acetate/hexane 1:9) afforded (E)-ethyl 3-(4-(tert-butyldimethylsilyloxy)phenyl) acrylate 5 (10.13 g, 92\%) as color less liquid. 
IR (neat): 2934, 1713, 1601, 1509, 1263, 1167, $912 \mathrm{~cm}^{-1} .{ }^{1} \mathrm{H}$ NMR $\left(300 \mathrm{MHz}, \mathrm{CDCl}_{3}\right): \delta 7.55$ (d, $J=15.8 \mathrm{~Hz}, 1 \mathrm{H}), 7.37$ (d, $J=8.3 \mathrm{~Hz}, 2 \mathrm{H}), 6.77(\mathrm{~d}, J=8.3 \mathrm{~Hz}, 2 \mathrm{H}), 6.21(\mathrm{~d}, J=15.8 \mathrm{~Hz}, 1 \mathrm{H}), 4.21$ (q, $J=7.5 \mathrm{~Hz}, 2 \mathrm{H}), 1.33(\mathrm{t}, J=7.5 \mathrm{~Hz}, 3 \mathrm{H}), 0.98(\mathrm{~s}, 9 \mathrm{H}), 0.21(\mathrm{~s}, 6 \mathrm{H})$. ESI-MS: $\mathrm{m} / \mathrm{z} 329$ [M+Na] $^{+}$. HRMS (ESI) $[\mathrm{M}+\mathrm{Na}]^{+} \mathrm{m} / \mathrm{z}$ Calcd for $\mathrm{C}_{17} \mathrm{H}_{26} \mathrm{O}_{3} \mathrm{NaSi}$ : 329.1548; found; 329.1544 .

\section{3-(4-(tert-Butyldimethylsilyloxy)phenyl)propionic acid ethyl ester}

A mixture of $5(8.2 \mathrm{~g}, 26.77 \mathrm{mmol}), 10 \% \mathrm{Pd} / \mathrm{C}$ in ethyl acetate $(75 \mathrm{~mL})$ was stirred at $25{ }^{0} \mathrm{C}$ under $\mathrm{H}_{2}(1 \mathrm{~atm})$ for $12 \mathrm{~h}$. After completion of reaction (monitored by TLC), it was filtered through a celite (ethyl acetate as eluent) and the solvent evaporated under vacuum to afford 3-(4(tert-butyldimethylsilyloxy)phenyl)propionic acid ethyl ester (7.50 g, 92\%) as colorless liquid.

IR (neat): 2934, 1736, 1510, 1258, 1169, $916 \mathrm{~cm}^{-1} .{ }^{1} \mathrm{H}$ NMR $\left(500 \mathrm{MHz}, \mathrm{CDCl}_{3}\right): \delta 6.99(\mathrm{~d}, J=$ $7.8 \mathrm{~Hz}, 2 \mathrm{H}), 6.68(\mathrm{~d}, J=7.8 \mathrm{~Hz}, 2 \mathrm{H}), 4.08(\mathrm{q}, J=6.8 \mathrm{~Hz}, 2 \mathrm{H}), 2.84(\mathrm{t}, J=7.8 \mathrm{~Hz}, 2 \mathrm{H}), 2.53(\mathrm{t}, J=$ $7.8 \mathrm{~Hz}, 2 \mathrm{H}), 1.22(\mathrm{t}, J=6.8 \mathrm{~Hz}, 3 \mathrm{H}), 0.97(\mathrm{~s}, 9 \mathrm{H}), 0.17(\mathrm{~s}, 6 \mathrm{H})$. ESI-MS: $m / z 309[\mathrm{M}+\mathrm{H}]^{+}$. HRMS (ESI) $[\mathrm{M}+\mathrm{Na}]^{+} \mathrm{m} / \mathrm{z}$ Calcd for $\mathrm{C}_{17} \mathrm{H}_{28} \mathrm{O}_{3} \mathrm{NaSi}$ : 331.1705; found; 331.1716 .

3-(4-(tert-Butyldimethylsilyloxy)phenyl)propan-1-ol (6). To a stirred suspension of $\mathrm{LiAlH}_{4}$ $(1.35 \mathrm{~g}, 35.55 \mathrm{mmol})$ in dry THF $(30 \mathrm{~mL})$, was added drop wise 3-(4-(tertbutyldimethylsilyloxy)phenyl)propionic acid ethyl ester (7.3 g, $23.70 \mathrm{mmol})$ in dry THF (40 mL) at $0{ }^{0} \mathrm{C}$. After $1.5 \mathrm{~h}$ at $0{ }^{0} \mathrm{C}$, it was quenched with the addition of saturated aqueous $\mathrm{Na}_{2} \mathrm{SO}_{4}$ solution, filtered through a celite pad (hot ethyl acetate as eluent). Residue obtained after evaporating the combined solvent under vacuum was purified by column chromatography (ethyl acetate/ hexane 15:85) to give 3-(4-(tert-butyldimethylsilyloxy)phenyl) propan-1-ol, 6 (5.73 g, 91\%) as colorless liquid. IR (neat): 3416, 2932, 1508, 1254, 1041, $914 \mathrm{~cm}^{-1}$. ${ }^{1} \mathrm{H}$ NMR (500MHz, $\left.\mathrm{CDCl}_{3}\right): \delta 6.98(\mathrm{~d}, J=7.8 \mathrm{~Hz}, 2 \mathrm{H}), 6.68(\mathrm{~d}, J=7.8 \mathrm{~Hz}, 2 \mathrm{H}), 3.65-3.60(\mathrm{~m}, 2 \mathrm{H}), 2.62(\mathrm{t}, J=7.8 \mathrm{~Hz}$, 2H), 1.86-1.80 (m, 2H), $0.97(\mathrm{~s}, 9 \mathrm{H}), 0.17(\mathrm{~s}, 6 \mathrm{H}) .{ }^{13} \mathrm{C} \mathrm{NMR}\left(75 \mathrm{MHz}, \mathrm{CDCl}_{3}\right): \delta 153.6,134.3$, $129.1,119.8,62.3,34.3,31.2,25.6,18.1$.

\section{3-(4-(tert-Butyldimethylsilyloxy)phenyl)propanal}

To a solution of oxalyl chloride $(16.9 \mathrm{mmol}, 1.41 \mathrm{~mL})$ in dry dichloromethane $(20 \mathrm{~mL})$ at $-78{ }^{\circ} \mathrm{C}$, DMSO (3.2 mL, $45.08 \mathrm{mmol})$ was added dropwise with stirring under nitrogen atmosphere. After $15 \mathrm{~min}$, compound 6 (3 g, $11.27 \mathrm{mmol}$ ) was added into the reaction mixture and subsequently after stirring for $30 \mathrm{~min}$ at $-78{ }^{\circ} \mathrm{C}, \mathrm{Et}_{3} \mathrm{~N}(56.35 \mathrm{mmol}, 7.8 \mathrm{~mL})$ was added and the mixture was stirred for another $0.5 \mathrm{~h}$ at $-78^{\circ} \mathrm{C}$ and then for $0.5 \mathrm{~h}$ at $0{ }^{\circ} \mathrm{C}$. The reaction mixture was quenched with saturated $\mathrm{NH}_{4} \mathrm{Cl}$ solution $(40 \mathrm{~mL})$ at $0{ }^{\circ} \mathrm{C}$ and extracted with EtOAc $(5 \times 30$ $\mathrm{mL}$ ). The combined organic extracts were washed with brine, dried over anhydrous $\mathrm{Na}_{2} \mathrm{SO}_{4}$ and concentrated in vacuum. The 3-(4-(tert-butyldimethylsilyloxy)phenyl)propanal thus obtained after flash column chromatography $(2.79 \mathrm{~g}, 94 \%)$ was used directly for further reaction.

${ }^{1} \mathrm{H}$ NMR $\left(300 \mathrm{MHz}, \mathrm{CDCl}_{3}\right): \delta 9.78(\mathrm{~s}, 1 \mathrm{H}), 6.98(\mathrm{~d}, J=8.7 \mathrm{~Hz}, 2 \mathrm{H}), 6.68(\mathrm{~d}, J=8.7 \mathrm{~Hz}, 2 \mathrm{H}), 2.86$ (t, $J=7.8 \mathrm{~Hz}, 2 \mathrm{H}), 2.71(\mathrm{t}, J=6.8 \mathrm{~Hz}, 2 \mathrm{H}), 0.97(\mathrm{~s}, 9 \mathrm{H}), 0.17(\mathrm{~s}, 6 \mathrm{H})$.

(E)-Ethyl 5-(4-(tert-butyldimethylsilyloxy)phenyl) pent-2-enoate (7). A solution of 3-(4-(tertbutyldimethylsilyloxy) phenyl) propanal (2.0 g, $7.57 \mathrm{mmol})$ and ethyl (triphenyl phosphor 
rnylidene) acetate $(3.84 \mathrm{~g}, 11.36 \mathrm{mmol})$ in benzene $(20 \mathrm{~mL})$, was refluxed for $8 \mathrm{~h}$. After completion of reaction (by TLC), it was concentrated in vacuum and the residue was purified by column chromatography (ethyl acetate/ hexane 5:95) to afford 7 (2.30 g, 91\%) as a colorless liquid. IR (neat): 2932, 1721, 1509, 1259, 1192, $915 \mathrm{~cm}^{-1} .{ }^{1} \mathrm{H}$ NMR (500 MHz, $\left.\mathrm{CDCl}_{3}\right): \delta 6.96$ $(\mathrm{d}, J=7.8 \mathrm{~Hz}, 2 \mathrm{H}), 6.91(\mathrm{~d}, J=15.6 \mathrm{~Hz}, 1 \mathrm{H}), 6.89(\mathrm{~d}, J=7.8 \mathrm{~Hz}, 2 \mathrm{H}), 5.76(\mathrm{~d}, J=15.6 \mathrm{~Hz}, 1 \mathrm{H})$, 4.14 (q, $J=6.8 \mathrm{~Hz}, 2 \mathrm{H}), 2.69$ (t, $J=7.8 \mathrm{~Hz}, 2 \mathrm{H}), 2.46$ (q, $J=7.8 \mathrm{~Hz}, 2 \mathrm{H}), 1.28(\mathrm{t}, J=6.8 \mathrm{~Hz}, 3 \mathrm{H})$, $0.97(\mathrm{~s}, 9 \mathrm{H}), 0.17(\mathrm{~s}, 6 \mathrm{H}) .{ }^{13} \mathrm{C} \mathrm{NMR}\left(75 \mathrm{MHz}, \mathrm{CDCl}_{3}\right): \delta 166.5,153.8,148.1,133.4,129.1$, 121.6, 119.9, 60.0, 34.0, 33.4, 25.4, 18.1, 14.1, -4.5. ESI-MS: m/z 335 [M+H] $]^{+}$HRMS (ESI) $[\mathrm{M}+\mathrm{Na}]^{+} \mathrm{m} / \mathrm{z}$ Calcd for $\mathrm{C}_{19} \mathrm{H}_{30} \mathrm{O}_{3} \mathrm{NaSi}$ : 357.1861; found: 357.1856 .

(2R,3S)-Ethyl 5-(4-(tert-butyldimethylsilyloxy)phenyl)-2,3-dihydroxypentanoate (8). To a solution of AD mix- $\alpha(4.2 \mathrm{~g})$ in $t-\mathrm{BuOH}: \mathrm{H}_{2} \mathrm{O}(1: 1,20 \mathrm{~mL})$, was added $\mathrm{MeSO}_{2} \mathrm{NH}_{2}(300 \mathrm{mg})$ and alkene $7(1.0 \mathrm{~g}, 2.99 \mathrm{mmol})$ slowly at $0^{0} \mathrm{C}$ and the mixture was stirred overnight at the same temperature. After complete consumption of the starting material, sodium sulfite $(4.5 \mathrm{~g})$ was added and the solution was warmed to room temperature and stirred for $90 \mathrm{~min}$. After which the reaction mixture was poured in water $(10 \mathrm{ml})$ and several times with ethyl acetate $(4 \times 100 \mathrm{~mL})$. Combined organic extracts were washed with brine $(2 \times 100 \mathrm{~mL})$, dried over $\mathrm{Na}_{2} \mathrm{SO}_{4}$ and concentrated in vacuum. Crude residue was chromato- graphed over silica (ethyl acetate/hexane 30:70) afforded $8(0.93 \mathrm{~g}, 85 \%)$ as colorless liquid. [ $\alpha]_{\mathrm{D}}{ }^{25}=-7.0$ (c 1.0, $\left.\mathrm{CHCl}_{3}\right)$.IR (neat): 3432, 2933, 2858, 1736, 1509, 1257, 1118, $916 \mathrm{~cm}^{-1} .{ }^{1} \mathrm{H}$ NMR $\left(300 \mathrm{MHz}, \mathrm{CDCl}_{3}\right.$ ): $\delta 7.00$ (d, J=8.3Hz, $2 \mathrm{H}), 6.68(\mathrm{~d}, J=8.3 \mathrm{~Hz}, 2 \mathrm{H}), 4.25(\mathrm{q}, J=7.1 \mathrm{~Hz}, 2 \mathrm{H}), 4.01-3.99(\mathrm{~m}, 1 \mathrm{H}), 3.85-3.77(\mathrm{~m}, 1 \mathrm{H})$, 3.01-2.95 (m, 1H), 2.79-2.57 (m, 2H), 1.92-1.79 (m, 3H), $1.31(\mathrm{t}, J=7.1 \mathrm{~Hz}, 3 \mathrm{H}), 0.97(\mathrm{~s}, 9 \mathrm{H})$, 0.17 (s, 6H). ${ }^{13} \mathrm{C}$ NMR $\left(75 \mathrm{MHz}, \mathrm{CDCl}_{3}\right): \delta 173.4,153.7,134.0,129.2,119.9,73.1,71.7,62.1$, 35.5, 31.0, 25.6, 18.1, 14.1, -4.4. ESI-MS: m/z $391[\mathrm{M}+\mathrm{Na}]^{+}$. HRMS (ESI) $[\mathrm{M}+\mathrm{Na}]^{+} \mathrm{m} / \mathrm{z}$ Calcd for $\mathrm{C}_{19} \mathrm{H}_{32} \mathrm{O}_{5} \mathrm{NaSi}$ : 391.1916; found: 391.1916 .

Cyclic sulfite, 9. To a solution of the compound $8(0.85 \mathrm{~g}, 2.30 \mathrm{mmol})$ in $\mathrm{CH}_{2} \mathrm{Cl}_{2}(20 \mathrm{~mL})$ at $0^{0} \mathrm{C}$, was added $\mathrm{Et}_{3} \mathrm{~N}(0.64 \mathrm{~mL}, 4.61 \mathrm{mmol})$ followed by freshly distilled thionyl chloride $(0.2$ $\mathrm{mL}, 2.77 \mathrm{mmol}$ ) drop wise. It was stirred further for $45 \mathrm{~min}$ at $0^{0} \mathrm{C}$ and then quenched by adding $\mathrm{H}_{2} \mathrm{O}(20 \mathrm{~mL})$. The phases were separated, aqueous phase was extracted with $\mathrm{CH}_{2} \mathrm{Cl}_{2}(3 \times 50$ $\mathrm{mL}$ ), combined organic phases were dried with $\mathrm{Na}_{2} \mathrm{SO}_{4}$ and concentrated in vacuum. After flash column chromatography this cyclic sulfite $9(0.89 \mathrm{~g}, 94 \%)$ was used for the next reaction.

Cyclic sulfate, 3. The cyclic sulfite $9(0.70 \mathrm{~g}, 1.69 \mathrm{mmol})$ dissolved in $\mathrm{CCl}_{4}(10 \mathrm{~mL})$ and $\mathrm{CH}_{3} \mathrm{CN}(10 \mathrm{~mL})$ was cooled in an ice bath and cold $\mathrm{H}_{2} \mathrm{O}(15 \mathrm{~mL})$ followed by $\mathrm{RuCl}_{3} \cdot \mathrm{H}_{2} \mathrm{O}(17$ $\mathrm{mg}, 0.10 \mathrm{mmol})$ and $\mathrm{NaIO}_{4}(0.72 \mathrm{~g}, 3.38 \mathrm{mmol})$ were added at once. The reaction mixture was vigorously stirred at $0^{0} \mathrm{C}$ for $30 \mathrm{~min}$, extracted with ether $(3 \times 50 \mathrm{~mL})$; the organic layer was washed with brine, dried over $\mathrm{Na}_{2} \mathrm{SO}_{4}$ and concentrated in vacuum. The crude residue was purified by column chromatography to afford $3(0.66 \mathrm{~g}, 92 \%)$ as violet color liquid. $[\alpha]_{\mathrm{D}}^{25}=-$ 46.2 (c 1.0, $\mathrm{CHCl}_{3}$ ).

IR (neat): 2933, 1768, 1510, 1398, 1259, 1209, 1018, $\left.912 \mathrm{~cm}^{-1} .{ }^{1} \mathrm{H} \mathrm{NMR} \mathrm{(500} \mathrm{MHz,} \mathrm{CDCl} \mathrm{Cl}_{3}\right): \delta$ $7.00(\mathrm{~d}, J=8.7 \mathrm{~Hz}, 2 \mathrm{H}), 6.72(\mathrm{~d}, J=7.8 \mathrm{~Hz}, 2 \mathrm{H}), 4.87-4.83(\mathrm{~m}, 1 \mathrm{H}), 4.75(\mathrm{~d}, J=7.8 \mathrm{~Hz}, 1 \mathrm{H}), 4.29$ $(\mathrm{q}, J=6.8 \mathrm{~Hz}, 2 \mathrm{H}), 2.87-2.82(\mathrm{~m}, 1 \mathrm{H}), 2.74-2.68(\mathrm{~m}, 1 \mathrm{H}), 2.30-2.19(\mathrm{~m}, 2 \mathrm{H}), 1.33(\mathrm{t}, J=6.8 \mathrm{~Hz}$, 
3H), 0.97 (s, 9H), $0.18(\mathrm{~s}, 6 \mathrm{H}) .{ }^{13} \mathrm{C}$ NMR $\left(75 \mathrm{MHz}, \mathrm{CDCl}_{3}\right): \delta=164.5,154.2,131.4,129.2$, 120.2, 83.0, 79.6, 63.1, 34.7, 30.0, 25.5, 18.0, 13.8, -4.5. ESI-MS: $\mathrm{m} / \mathrm{z} 453[\mathrm{M}+\mathrm{Na}]^{+}$. HRMS (ESI) $[\mathrm{M}+\mathrm{Na}]^{+} \mathrm{m} / \mathrm{z}$ Calcd for $\mathrm{C}_{19} \mathrm{H}_{30} \mathrm{O}_{7} \mathrm{NaSi}$ : 453.1379; found: 453.1361 .

(S)-Ethyl 5-(4-(tert-butyldimethylsilyloxy)phenyl) -3-hydroxypentanoate (10). To a stirred solution of cyclic sulfate $3(0.30 \mathrm{~g}, 0.69 \mathrm{mmol})$ in dry DMF $(5 \mathrm{~mL})$ at $0{ }^{\circ} \mathrm{C}$, was added $\mathrm{NaBH}_{4}$ (52 mg, $1.39 \mathrm{mmol}$ ) under $\mathrm{N}_{2}$ atm. After $15 \mathrm{~min}$ of stirring, solvent was removed under reduced pressure $\left(0.2 \mathrm{~mm} \mathrm{Hg}\right.$, ) and the residue was suspended in dry THF $(10 \mathrm{~mL})$. At $0^{0} \mathrm{C}$, conc. $\mathrm{H}_{2} \mathrm{SO}_{4}$ $(35 \mu \mathrm{L})$ and $\mathrm{H}_{2} \mathrm{O}(15 \mu \mathrm{L})$ were added slowly with stirring for another 20 min and excess sodium bicarbonate $(200 \mathrm{mg})$ was added with stirring further for $20 \mathrm{~min}$. Filtered through a celite pad (hot ethyl acetate as eluent), filtrate was concentrated under reduced pressure and the crude liquid was chromatographed (ethyl acetate/ hexane 20:80) to give (S)-ethyl5-(4-(tertbutyldimethylsilyloxy)phenyl)-3-hydroxypentanoate $10(0.22 \mathrm{~g}, 90 \%)$ as a colorless liquid. $[\alpha]$ $\mathrm{D}^{25}=-5.0\left(\mathrm{c} 1.0, \mathrm{CHCl}_{3}\right)$. IR (neat): 3451, 2931, 2858, 1727, 1509, 1256, 1179, $915 \mathrm{~cm}^{-1} .{ }^{1} \mathrm{H}$ NMR $\left(500 \mathrm{MHz}, \mathrm{CDCl}_{3}\right): \delta 6.99(\mathrm{~d}, J=8.1 \mathrm{~Hz}, 2 \mathrm{H}), 6.67(\mathrm{~d}, J=8.1 \mathrm{~Hz}, 2 \mathrm{H}), 4.14(\mathrm{q}, J=7.2 \mathrm{~Hz}$, 2H), 3.96-3.91 (m, 1H), $2.94(\mathrm{~d}, J=3.6 \mathrm{~Hz}, 1 \mathrm{H}), 2.75-2.69(\mathrm{~m}, 1 \mathrm{H}), 2.63-2.57(\mathrm{~m}, 1 \mathrm{H}), 2.46-2.35$ (m, 2H), 1.81-1.74 (m, 1H), 1.68-1.62 (m, 1H), 1.27 (d, J=7.2Hz, 3H), 0.97 (s, 9H), 0.17 (s, $6 \mathrm{H}) .{ }^{13} \mathrm{C}$ NMR $\left(75 \mathrm{MHz}, \mathrm{CDCl}_{3}\right): \delta 172.9,153.6,134.2,129.1,119.8,67.1,60.6,41.2,38.2,30.8$, 29.6, 25.6, 18.1, 14.1, -4.4.ESI-MS: $\mathrm{m} / \mathrm{z} 353[\mathrm{M}+\mathrm{H}]^{+}$. HRMS (ESI) $[\mathrm{M}+\mathrm{Na}]^{+} \mathrm{m} / \mathrm{z}$ Calcd for $\mathrm{C}_{19} \mathrm{H}_{32} \mathrm{O}_{4} \mathrm{NaSi}$ : 375.1967 ; found: 375.1981 .

(S)-Ethyl 3-(tert-butyldimethylsilyloxy)-5-(4-(tert-butyldimethylsilyloxy)phenylpentanoate (11). To compound $10(0.15 \mathrm{~g}, 0.42 \mathrm{mmol})$ in dry $\mathrm{CH}_{2} \mathrm{Cl}_{2}(5 \mathrm{~mL})$, imidazole ( $\left.86 \mathrm{mg}, 1.27 \mathrm{mmol}\right)$ followed by TBS-Cl $(130 \mathrm{mg}, 0.85 \mathrm{mmol})$ were added at $0{ }^{\circ} \mathrm{C}$ under $\mathrm{N}_{2}$. After stirring the reaction mixture over night, it was quenched with a saturated solution of $\mathrm{NH}_{4} \mathrm{Cl}(3 \mathrm{~mL})$ and extracted with $\mathrm{CH}_{2} \mathrm{Cl}_{2}(6 \times 25 \mathrm{~mL})$. The combined organic extracts were washed with brine, dried over anhydrous $\mathrm{Na}_{2} \mathrm{SO}_{4}$ and concentrated in vacuum. The crude residue was flash chromatographed (ethyl acetate/hexane 5:95) to afford (S)-ethyl 3-(tert-butyldimethylsilyloxy)5-(4-(tert-butyl dimethylsilyloxy)phenyl)pentanoate $\mathbf{1 1}(0.18 \mathrm{~g}, 95 \%)$ as colorless liquid. [ $\alpha]_{\mathrm{D}}{ }^{25}=$ +10.6 (c 1.0, $\left.\mathrm{CHCl}_{3}\right)$.IR (neat): 2932, 2858, 1737, 1509, 1256, 1091, $916 \mathrm{~cm}^{-1} .{ }^{1} \mathrm{H}$ NMR $\left(300 \mathrm{MHz}, \mathrm{CDCl}_{3}\right.$ ): $\delta 7.00(\mathrm{~d}, J=8.3 \mathrm{~Hz}, 2 \mathrm{H}), 6.73(\mathrm{~d}, J=8.3 \mathrm{~Hz}, 2 \mathrm{H}), 4.20-4.08(\mathrm{~m}, 3 \mathrm{H}), 2.62-$ $2.52(\mathrm{~m}, 2 \mathrm{H}), 2.47(\mathrm{t}, J=6.7 \mathrm{~Hz}, 2 \mathrm{H}), 1.82-1.73(\mathrm{~m}, 2 \mathrm{H}), 1.25(\mathrm{t}, J=6.7 \mathrm{~Hz}, 3 \mathrm{H}), 0.97(\mathrm{~s}, 9 \mathrm{H})$, 0.88 (s, 9H), 0.17 (s, 6H), $0.06(\mathrm{~s}, 3 \mathrm{H}), 0.03(\mathrm{~s}, 3 \mathrm{H})$.

${ }^{13} \mathrm{C}$ NMR $\left(75 \mathrm{MHz}, \mathrm{CDCl}_{3}\right): \delta 171.7,153.5,134.6,129.0,119.8,68.9,60.3,42.5,39.4,30.4$, 25.7, 25.6, 18.1, 14.1, -4.8, -4.5, -4.4.ESI-MS: $\mathrm{m} / \mathrm{z} 467[\mathrm{M}+\mathrm{H}]^{+}$. HRMS (ESI) $[\mathrm{M}+\mathrm{Na}]^{+} \mathrm{m} / \mathrm{z}$ Calcd for $\mathrm{C}_{25} \mathrm{H}_{46} \mathrm{O}_{4} \mathrm{NaSi}_{2}$ : 489.2832; found: 489.2853 .

(S)-3-(tert-butyldimethylsilyloxy)-5-(4-(tert-butyl dimethylsilyloxy) phenyl)pentanal (2). To a solution of (S)-ethyl 3-(tert-butyldimethylsilyloxy)-5-(4-(tert-butyldimethylsilyloxy)phenyl pentanoate $11(0.1 \mathrm{~g}, 0.21 \mathrm{mmol})$ in dry $\mathrm{CH}_{2} \mathrm{Cl}_{2}(2 \mathrm{~mL})$, DIBAL-H $(1.0 \mathrm{M}, 0.23 \mathrm{~mL}, 0.23 \mathrm{mmol})$ was added drop wise at $-78{ }^{\circ} \mathrm{C}$ and stirred for $10 \mathrm{~min}$. The reaction mixture was quenched with saturated aqueous sodium potassium tartrate $(1 \mathrm{~mL})$ at $-78{ }^{\circ} \mathrm{C}$, stirred for $20 \mathrm{~min}$ and then brought to room temperature. The organic layer was separated and aqueous layer was extracted 
with EtOAC $(4 \times 10 \mathrm{~mL})$, combined organic extracts were dried over anhydrous $\mathrm{Na}_{2} \mathrm{SO}_{4}$ and concentrated in vacuum. The crude residue thus obtained was purified by column chromatography (ethyl acetate/hexane 10:90) to afford (S)-3-(tert-butyldimethylsilyloxy)-5-(4(tert-butyldimethyl silyloxy)phenyl)pentanal $2(0.078 \mathrm{~g}, 88 \%)$ as a colorless liquid. $[\alpha]_{\mathrm{D}}{ }^{25}=+$ 5.54 (c 2.1, $\left.\left.\mathrm{CHCl}_{3}\right) ; \mathrm{Lit}^{3 \mathrm{c}}[\alpha]_{\mathrm{D}}{ }^{25}=+5.57\right) .{ }^{1} \mathrm{H} \mathrm{NMR}\left(500 \mathrm{MHz}, \mathrm{CDCl}_{3}\right): \delta 9.80(\mathrm{t}, J=1.9 \mathrm{~Hz}, 1 \mathrm{H})$, $6.99(\mathrm{~d}, J=8.7 \mathrm{~Hz}, 2 \mathrm{H}), 6.73(\mathrm{~d}, J=8.7 \mathrm{~Hz}, 2 \mathrm{H}), 4.22(\mathrm{q}, J=5.8 \mathrm{~Hz}, 1 \mathrm{H}), 2.60-2.55(\mathrm{~m}, 4 \mathrm{H}), 1.85-$ $1.80(\mathrm{~m}, 2 \mathrm{H}), 0.98$ (s, 9H), 0.89 (s, 9H), $0.18(\mathrm{~s}, 6 \mathrm{H}), 0.07$ (s, 3H), $0.04(\mathrm{~s}, 3 \mathrm{H}) .{ }^{13} \mathrm{C}$ NMR $\left(75 \mathrm{MHz}, \mathrm{CDCl}_{3}\right): \delta 202.0,153.7,134.2,129.0,119.9,67.7,50.7,39.6,30.6,25.7,18.1,17.9$, 4.4, -4.6.

\section{Acknowledgments}

We thank Dr. J. S. Yadav, Director IICT, and Dr. V.V. Narayana Reddy, Head, Organic Chemistry Division- II for their constant encouragement and support. S.V.N.V. (SRF) and S.R.P. (JRF) thank CSIR, New Delhi for the award of fellowships.

\section{Supplementary Material}

Copies of ${ }^{1} \mathrm{H}$ NMR and ${ }^{13} \mathrm{C}$ NMR of compounds 2, 3, 7, 8, 10 and 11.

\section{References}

1. Ouedraogo, M.; Carreyre, H.; Vandelrouck, C.; Bescound, J.; Raymond, G.; Guissou, I.-P.; Cognard, C.; Becq, F.; Potreau, D.; Cousson, A.; Marrot, J.; Coustard, J.-M. J. Nat. Prod. 2007, 70, 2006.

2. (a) Jodynis-Liebert, J.; Murias, M.; Bloszyk, E. Planta Med. 2000, 66, 199. (b) Nicolaou, K. C.; Rodriguez, R. M.; Mitchell, H. J.; Suzuki, H.; Fylaktakidou, K. C.; Baudoin, O.; van Delft, F. L. Chem. Eur. J. 2000, 6, 3095. (c) Carda, M.; Gonzalez, F.; Castillo, E.; Rodriguez, S.; Marco, J. A. Eur. J. Org. Chem. 2002, 2649. (d) Murga, J.; Falomir, E.; Garcia-Fortanet, J.; Carda, M.; Marco, J. A. Org. Lett. 2002, 4, 3447. (e) Bouzbouz, S.; Cossy, J. Org. Lett. 2003, 5, 1995. (f) Sabitha, G.; Bhaskar, V.; Reddy, S. S. S.; Yadav, J. S. Tetrahedron 2008, 64, 10207.

3. (a)Alvarez-Bercedo, P.; Falomir, E.; Murga, J.; Carda, M.; Marco, J. A. Eur. J. Org. Chem. 2008, 4015. (b) Dittoo, A.; Bellosta, V.; Cossy, J. Synlett 2008, 16, 2459. (c) Rauniyar, V.; Hall, D. G. J. Org. Chem. 2009, 74, 4236.

4. Srihari, P.; Rajender, G.; Rao, R.S.; Yadav, J. S. Tetrahedron Lett. 2008, 49, 5590. 
5. Das, B.; Suneel, K.; Satyalakshmi, G.; Kumar, D. N. Tetrahedron: Asymmetry 2009, 20, 13, 1536.

6. (a) Byun, H-S.; He, L.; Bittman R. Tetrahedron 2000, 56, 7051. (b) Qin, D-G.; Zha, H-Y.; Yao, Z-J. J.Org. Chem. 2002, 67, 1038. (c) Fernandes, R. A.; Kumar, P. Eur. J. Org. Chem. 2002, 2921. (d) Gupta, P.; Naidu, S. V.; Kumar, P. Tetrahedron Lett. 2004, 45, 9641. (e) Jagdale, A. R.; Reddy, Sudalai, A. Org. Lett. 2009, 11, 803.

7. (a) Gao, Y.; Sharpless, K. B. J. Am. Chem. Soc., 1988, 110, 7538. (b) Lohray, B. B. Synthesis, 1992, 1035.

8. Kim, B. M.; Sharpless, K. B. Tetrahedron Lett. 1989, 6, 655. 